\title{
Public understanding of medical terminology: non-English speakers may not receive optimal care
}

\author{
M W Cooke, S Wilson, P Cox, A Roalfe
}

\begin{abstract}
Introduction-Many systems of telephone triage are being developed (including NHS Direct, general practitioner out of hours centres, ambulance services). These rely on the ability to determine key facts from the caller. Level of consciousness is an important indicator after head injury but also an indicator of severe illness.

Aims-To determine the general public's understanding of the term unconscious. Methods-A total of 700 people were asked one of seven questions relating to their understanding of the term unconscious. All participants were adults who could speak sufficient English to give a history to a nurse.

Results-Correct understanding of the term unconscious varied from $46.5 \%$ to $\mathbf{8 7 . 0 \%}$ for varying parameters. Those with English as their first language had a better understanding $(\mathbf{p}<0.01)$ and there was a significant variation with ethnicity $(\mathbf{p}<0.05)$.

Conclusions-Understanding of the term unconscious is poor and worse in those for whom English is not a first language. Decision making should not rely on the interpretation of questions using technical terms such as unconscious, which may have a different meaning between professional and lay people.

(f Accid Emerg Med 2000;17:119-121)
\end{abstract}

Keywords: medical terminology; triage; communication

University of Warwick and Walsgrave

Hospitals NHS Trust,

Coventry

$\mathrm{M}$ W Cooke

Emergency Medicine Research Group, Department of

General Practice and

Primary Care,

University of

Birmingham

$\mathrm{S}$ Wilson

A Roalfe

City Hospital NHS

Trust, Birmingham

P Cox

Correspondence to:

Dr Matthew Cooke, Senior

Lecturer in Emergency Care, Emergency Medicine

Research Group, Primary

Care Unit, University of

Warwick, Coventry CV4 7AL

(e-mail: MWCooke@

emerg-uk.com)

Accepted 28 September 1999

Table 1 Questions relating to the term unconscious preferences. ${ }^{23}$ Medical terms have been shown to be widely misinterpreted by the public, ${ }^{4}$ including a perceived difference between the terms fracture and a break. ${ }^{5}$ Further studies suggest that patients and doctors refer to different entities when they talk of constipation $^{6}$ and back pain. ${ }^{7}$

If patients misunderstand the term unconscious it can have serious implications. Decisions on radiography and admitting patients are partly dependent on information regarding loss of consciousness. However, the importance of the public's understanding of medical terminology has recently been extended as some ambulance services have now introduced criteria based dispatch systems. These systems aim to triage emergency 999 calls and loss of consciousness is one of the criteria used to determine a rapid ambulance response. ${ }^{8}$ At least 3\% of 999 calls have communication problems caused by a lack of comprehension of medical terms. $^{9}$

The aim of this study was to assess whether the general public understood the term "unconscious".

\section{Methods}

For the purposes of this study unconsciousness was taken to mean the "state of being insensible or without conscious experiences .....patient is unable to swallow; eyes do not react and patient is insensible to surroundings" ${ }^{10}$ It was intended to ask 700 ambulant patients attending an inner city $\mathrm{A} \& \mathrm{E}$ department, during the period 29 June 1998 to 29 July 1998, one of seven questions relating to the term unconsciousness (table 1). For each patient a question was randomly selected from the list of seven questions being studied. The questioner was blinded to any analysis. The person asked the question was the one giving the history to the triage nurse. The rationale for asking this person is that this is the person on whom staff will rely for information. The person answering the question was also asked to state their ethnic origin and their first language.

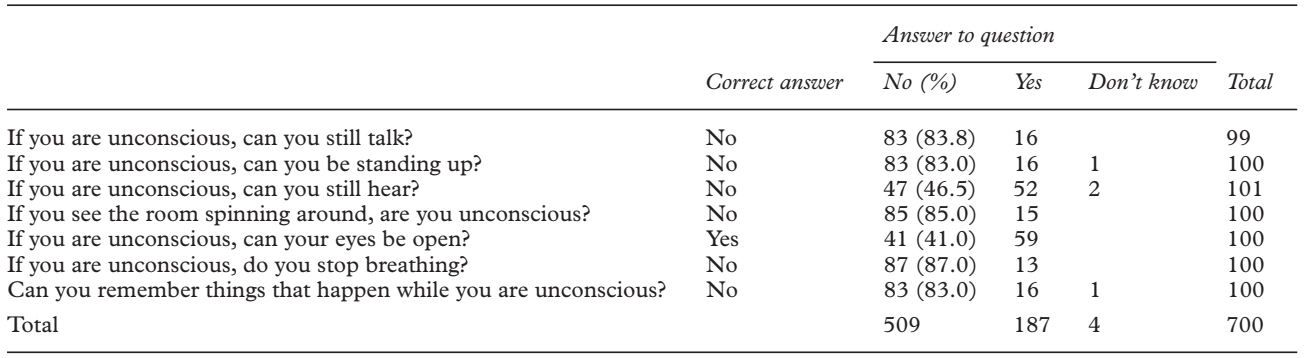


Table 2 Ethnic origin and whether English was first language

\begin{tabular}{llll}
\hline & \multicolumn{2}{l}{ English as first language } & \multicolumn{1}{l}{ Total No } \\
\cline { 2 - 3 } Ethnic origin & Yes (\%) & No (\%) & (\%) \\
\hline Afro-Caribbean & $102(98.1)$ & $2(1.9)$ & $104(14.9)$ \\
Asian & $65(37.6)$ & $108(62.4)$ & $173(24.7)$ \\
White & $403(99.0)$ & $4(1.0)$ & $407(58.1)$ \\
Other & $12(75.0)$ & $4(25.0)$ & $16(2.3)$ \\
Total & $582(83.1)$ & $118(16.9)$ & 700 \\
\hline
\end{tabular}

All patients were eligible to participate in the study except those who were unable to provide comprehensible information for nurse triage (whether because of language, mental ability, or alcohol), patients with head injuries or decreased conscious level, and those where any delay in treatment could be prejudicial. Patients under the age of 17 years were also excluded.

All data were entered on to an Excel spreadsheet and analysis was undertaken using SAS. Tests of association, allowing for different questions asked, were carried out using Mantel-Haenszel tests. ${ }^{10}{ }^{11}$ The $\chi^{2}$ and Fisher's exact tests with Bonferroni corrections were employed where appropriate. Logistic regression analysis with backward elimination was undertaken to establish the predictors of correct answers.

\section{Results}

Altogether 721 people were approached of whom 21 were excluded as neither they nor the person accompanying them spoke sufficient English to answer the question. Data were collected from 700 patients, each of whom answered one of the seven questions about the term unconscious. No patients refused to participate in the study.

The study population was primarily white $(\mathrm{n}=407,58 \%)$ but with a high proportion of Asians $(\mathrm{n}=173,25 \%)$ and Afro-Caribbeans $(\mathrm{n}=104,15 \%)$. The majority of participants reported English to be their first language $(\mathrm{n}=582,83 \%)($ table 2$)$.

In only four cases $(0.6 \%)$ did a person state that they did not know the answer to a question; these responses have been classified as incorrect answers in all further analyses (table 1). When combining the responses to all questions, $527(75.3 \%)$ of participants provided the "correct" answer, ranging from $46.5 \%$ (If you are unconscious, can you still hear?) to $87.0 \%$ (If you are unconscious, do

Table 3 Ethnic origin, whether English was first language, and "correct" answers

\begin{tabular}{llll}
\hline & \multicolumn{2}{c}{ Correct answer } & Total No \\
\cline { 2 - 3 } & Yes (\%) & No (\%) & \multicolumn{1}{c}{$(\%)$} \\
\hline Ethnic origin & & & \\
$\quad$ Afro-Caribbean & $78(75.0)$ & $26(25.0)$ & $104(14.9)$ \\
Asian & $115(66.5)$ & $58(33.5)$ & $173(24.7)$ \\
White & $321(78.9)$ & $86(21.1)$ & $407(58.1)$ \\
Other & $13(81.3)$ & $3(18.8)$ & $16(2.3)$ \\
Total & $527(75.3)$ & $173(24.7)$ & 700 \\
English as a first language & & \\
$\quad$ Yes & $453(77.8)$ & $129(22.2)$ & $582(83.1)$ \\
$\quad$ No & $74(62.7)$ & $44(37.3)$ & $118(16.9)$ \\
Total & $527(75.3)$ & $173(24.7)$ & 700 \\
\hline
\end{tabular}

you stop breathing?). The only two questions that did not elicit correct responses from more than $80 \%$ of participants were those related to hearing and eyes being open.

The proportion of correct responses ranged from $78.9 \%$ for whites, through $75.0 \%$ for Afro-Caribbeans, to $66.5 \%$ for Asians (table 3). A greater proportion of correct answers was given by respondents with English as a first language compared with the non-native English speakers $(77.8 \%$ v $62.7 \%)$.

After controlling for different questions there was evidence of an association between correct responses and ethnicity $\left(\chi_{\mathrm{MH}(3 \mathrm{df})}^{2}=10.3\right.$, $\mathrm{p}<0.05)$; and also between correct responses and English as a first language $\left(\chi_{\mathrm{MH}(\mathrm{dd})}^{2}=13.8\right.$, $\mathrm{p}<0.01)$. Analyses by individual questions identified an association between correct responses to stopped breathing and ethnic group $(\mathrm{p}<0.05)$ and between correct responses to room spinning round with English as a first language (Fisher's exact test, $\mathrm{p}<0.02$ ). When the inter-relationships of all variables were examined via logistic regression analysis, the independent predictor of a correct answer was having English as a first language (odds ratio $2.4,95 \%$ confidence interval 1.5 to 3.7 ) as opposed to ethnicity.

\section{LIMITATIONS}

This study was undertaken in an A\&E department based in an urban area with a high proportion of patients from ethnic minorities and the findings may not, therefore, be as relevant to other areas. The findings are, however, likely to be generalisable to non-native English speakers in all areas of the UK.

In the UK the term "English as a second language" covers a very wide range of English language competence (and levels of education). Unfortunately it was outwith the remit of this study to explore this in any detail.

This study was not designed to establish whether there are other medical terms that are not widely understood by non-native English speakers or the reasons for the observed misunderstandings. It is possible that the translation equivalents of "is unconscious" in the respondent's first language could be phrases such as "can't stand, hear etc" or "feels faint". Further research should investigate the reasons for incorrect responses and examine other medical terms that affect the investigation and care of patients.

It is possible that while unconscious patients cannot react to noise they may still hear and such thinking may have affected responses to the question about hearing. We, therefore, also undertook analyses based on the six remaining questions alone ( $n=599$ respondents). The exclusion of the question relating to hearing raised the overall proportion of patients providing a correct answer from $75.3 \%$ to $80.1 \%$ but did not effect any of the conclusions or the significance of the findings.

\section{Discussion}

Those who do not have English as their first language may receive a lower priority of ambulance response because of their misunder- 
standing of a common medical term. In the $A \& E$ department they may be triaged for a longer wait, not receive necessary radiography, or fail to be admitted for observation because their misunderstanding results in them stating a person was not unconscious. Alternatively they may be over investigated and over utilise resources because of misunderstanding that labels someone unconscious when they are not.

Approximately a quarter of the population surveyed could not provide a correct response to questions aimed at eliciting their understanding of the term unconscious. Understanding of the term unconscious was lowest among those stating themselves to be of Asian origin, this being the population with the highest proportion (62\%) who did not have English as a first language. This study highlights the importance not only of achieving an answer to a question but also ensuring that any medical terms such as unconscious are understood. The value of asking supplementary questions, to establish whether a patient has been unconscious, has been demonstrated by this study. The questions relating to talking, standing, and remembering are those that cause least confusion and we recommend that ambulance service call takers and A\&E staff consider incorporating these questions into routine use.

Systems utilising information from patients must ensure they do not introduce discrepancies in health care because of a lack of comprehension of medical terms by non-English speaking individuals. Good translation facilities either with interpreters or visual aids such as descriptive cartoons may assist this process. Key questions, such as the presence of unconsciousness, should be verified with a supplementary question. This may include asking for a description of how the patient was acting or what the person means by the term.
As with any key decision, it should not rely on a single piece of evidence, which may be incorrect. Patient comprehension of medical terms should not be presumed to be correct simply because they provide a response.

Our thanks to Pam Bridge who meticulously undertook the patient interviews. John Skelton was kind enough to read and comment on the first draft of this paper.

Contributors

SW, PC, and MWC designed the study PC had the original idea, reviewed the literature, and undertook a pilot study. SW and AR undertook the statistical analysis. MWC undertook the initial study design, supervised data collection, and wrote the original draft of the paper. All the authors were involved in writing the paper.

MWC is the guarantor of the paper.

Conflict of interest: none.

Funding: none.

Ethics: approved by West Birmingham Research Ethics Committee.

1 Manchester Triage Group. Emergency triage. MackwayJones K, ed. London: BMJ Publishing Group, 1997.

2 Haidet $\mathrm{P}$, Hamel MB, Davis RB, et al. Outcomes, preferences for resuscitation and physician-patient communication among patients with metastatic colorectal cancer. Am ₹ Med 1998;105:222-9.

3 Mayeaux EJ Jr, Murphy PW, Arnold C, et al. Improving patient education for patients with low literacy skills. Am Fam Physician 1996;53:205-11.

4 Boyle CM. Difference between patients' and doctors' interpretation of some common medical terms. BMF 1970; i: $286-9$.

5 Peckham TJ. Doctor, have I got a fracture or a break? Injury 1994;25:221-2.

6 Herz MJ, Kahan S, Aframian R, et al. Constipation: a different entity for patients and doctors. Fam Pract 1996;13:1569.

7 Cedraschi C, Reust P, Roux E, et al. The role of prior knowledge on back-pain education. Fournal of Spinal Disorders 1992;5:267-76.

8 Cooke MW, Wilson S, Allan T, et al. Safety and effectiveness of criteria based dispatch in the prioritisation of 999 calls. of criteria based dispatch in the prioritisation of 999 calls. Birmingham: Emergency Mediti
versity of Birmingham, 1998.

9 Cooke MW, Wilson S. Are 999 callers in a position to give triage information and receive first aid advice? Pre-hospital Immediate Care 1998;2:193-6.

10 Taber CL. Taber's cyclopedic medical dictionary. 16th Ed. Philadelphia: F A Davis, 1989.

11 Armitage P, Berry G. Statistical methods in medical research. Oxford: Blackwell Scientific, 1994. 